\title{
La entrada del arte chino contemporáneo en Occidente. Inside Out: New Chinese Art, un caso de estudio
} The entry of contemporary Chinese art in the West. Inside Out: New Chinese Art, a case study

Ane Lekuona Mariscal Departamento de Historia del Arte, Universidad del País Vasco, España (anelekuona@gmail.com)

Recibido el 20 de diciembre de 2017; revisado el 10 de enero de 2018; aceptado el 18 de febrero de 2018; publicado el 21 de marzo de 2018

RESUMEN: El presente texto tiene como objetivo realizar un estudio sobre la exposición Inside Out: New Chinese Art celebrada entre los años 1998-1999 en Nueva York. Y es que al tomar la muestra como caso de estudio, es posible profundizar en torno a la entrada del arte contemporáneo chino en Occidente, fundamentalmente a lo largo de la década de los noventa. Diferentes factores como el momento histórico, el contexto socio-político, los intereses económicos, el novedoso discurso expositivo y su trascendencia para las siguientes exhibiciones de temática similar hicieron que la muestra se considere, actualmente, un acontecimiento significativo para la historia del arte chino contemporáneo y su introducción en Occidente.

PALABRAS CLAVE: Occidente, Oriente, exposiciones de Arte.

\begin{abstract}
The present text has the goal to fulfill a study about the exhibition Inside Out: New Chinese Art celebrated in New York between 1998-1999. Taking the exhibition as a case of study is possible to deep around the entrance of the Contemporary Chinese Art in Western, essentially throughout the 90s. Different factors must be taken into account, such as the historic time, the sociopolitical context, the economic interests, the innovative discourse of the show or its transcendence for the followings shows. Those elements have driven the exhibition as a significant event within the Contemporary Chinese Art History and its entrance in Western.
\end{abstract}

KEYWORDS: West, East, Art exhibitions. 


\section{Introducción}

En primer lugar, es necesario contextualizar el modo en el que el arte contemporáneo o experimental ${ }^{1}$ chino entró o se presentó en el marco y mercado occidental, generalmente en Europa, Estados Unidos, Canadá y Australia, y el porqué de tal interés repentino y cantidad de exposiciones sobre el panorama artístico que se llevaba a cabo en la República China.

El hecho de que China ganara tanta atención a escala mundial se debe ligar a los cambios socioculturales que vivió el país con el final de la Guerra Fría (1989). De ahí que en el proceso de apertura que estaba iniciando el gobierno de la República China, la proyección de su imagen en el resto de países occidentales capitalistas se convirtiese en una tarea fundamental, entrando en juego diferentes cuestiones como el proceso de modernización o la identidad cultural. La idea que se pretendía reforzar era la de una China que fuera el "amanecer de una nueva modernidad" o la de una nación que luchaba contra la tradición, en pro de una apertura al nuevo modelo económico y cultural. Asimismo, a esta auto-definida modernidad que del mismo modo, se percibía desde fuera, se sumaba el enfrentado deseo del propio gobierno chino por fortalecer y proteger su esencia nacional en resistencia al impacto cultural de Occidente. Por tanto, el país se encontraba en un proceso de modernidad a la "defensiva", el cual impactó profundamente en las identidades y subjetividades de la comunidad y también en el ámbito del arte y los artistas (Gao, 1998, pp. 15-40).

Así pues, en esta nueva etapa aperturista, las actividades relacionadas con el arte, como las diferentes exposiciones, ferias y muestras se convirtieron en otra herramienta diplomática para lograr la afiliación política entre la República de China y el horizonte internacional de Occidente. No obstante, siguiendo este proceso que se inició en la década de los noventa, para la entrada del nuevo siglo XXI, el arte contemporáneo chino y sus diferentes agentes - críticos, comisarios, artistas o coleccionistas - habían encontrado su lugar en el circuito internacional del arte (Erickson, 2002, p.107).

\section{El nuevo encuentro de Occidente con el arte chino.}

Anticipando el análisis de la exposición Inside Out: New Chinese Art es interesante realizar un breve registro sobre los diferentes hechos destacados en la llegada del arte experimental chino a Occidente.

\footnotetext{
${ }^{1}$ La terminología ha sido un debate recurrente a la hora de analizar el arte contemporáneo de China, siendo difícil establecer traducciones literales validas de la lengua china al inglés. Para más información consultar: GLADSTON, Paul (2014). Contemporary Chinese Art: A Critical History. Reaktion Books: Islington.
} 
Según los especialistas, en esta irrupción es posible diferenciar dos etapas o fases. La "primera ola de interés" sobre el arte experimental chino en el panorama internacional, o mejor dicho occidental, se dio a finales de la década de los ochenta e inicios de los noventa. En Estados Unidos se podría citar la exhibición Painting the Chinese Dream: Chinese Art Thirty Years after the Revolution, en la Smith College Museum of Art, en 1982 y Artist from China-New Expressions en la Sarah Lawrence College como muestras pioneras. En Europa, fue la exposición Magiciens de la Terre en el Centre Georges Pompidou en 1989 la que inició esta tendencia (Erickson, 2002, p.105). En estas primeras exposiciones, tanto en los textos de los catálogos como en el criterio de obras de arte seleccionadas, era visible el discurso orientalista, esto es, el hecho de presentar el arte de China como un material subalterno nooccidental. Precisamente, el título escogido para la ambiciosa exhibición de París hacía evidente la imagen exótica y mitificada que se pretendía trasladar del arte contemporáneo chino al público occidental $^{2}$.

Siguiendo esta sucesión, en el año 1993, teniendo en cuenta la relevancia que tendrían para la posterioridad, se dieron acontecimientos importantes, tales como las exposiciones China AvantGarde: Counter Currents in Art and Culture ${ }^{3}$, China's New Art, Post-19894 y Mao Goes Pop: China Post- $1989{ }^{5}$ y por último el hecho de que por primera vez diferentes artistas chinos fueron representados en la Biennale de Venecia ${ }^{6}$. Todas las exhibiciones citadas tenían como característica común el énfasis en la fecha 1989, señalando y canonizando este año como fecha clave para la historia del arte contemporáneo chino. Su razón se debía a que fue entonces, por un lado, se presentó la exposición China/Avant-garde ${ }^{7}$ en la National Gallery of Art de Beijing ${ }^{8}$, y por otro, se vivió el trágico final del movimiento o revuelta estudiantil en la plaza de Tin'anmen de la capital. A pesar de que las protestas tenían como origen las oraciones en honor a la muerte de Hu Yaobang, ex Secretario General del Partido Comunista expulsado del gobierno en 1987, su trasfondo se debe ligar

\footnotetext{
${ }^{2}$ Para más información consultar: CERVIÑO, Mariana Eva (2015), "Desigualdad y diferencia en el discurso del arte global”, Estudios sobre las Culturas Contemporáneas, vol. 21, no 41, pp. 9-39.

${ }_{3}^{3}$ Celebrada en Berlín en el Haus der Kulturen der Welt Berlin.

${ }^{4}$ Presentada en el Hong Kong Arts Centre en febrero.

${ }^{5}$ En el Museum of Contemporary Art en Sydney entre junio y agosto.

${ }^{6}$ Parar más información consultar: LAGO, Francesca (2000), “Chinese Art at the Venice Biennale: 1.”, en CLARK, John (ed.). Chinese Art at the end of the Millennium. Hong Kong: New Art Media Limited, pp. 158-166.

${ }^{7}$ Para más información consultar: KOCH, Franziska (2011), “'China' on Display for European Audiences? The Making of an Early Travelling Exhibition of Contemporary Chinese Art: China Avantgarde (Berlin/1993)”, Transcultural Studies, 2011, nº 2, pp. 66-139.

${ }^{8}$ Después de años de trabajo y siendo la primera gran muestra sobre arte contemporáneo chino organizado por los propios artistas chinos, la muestra se inauguró el 5 de febrero a las 9:00 de la mañana. exponiendo unas 300 obras de 186 artistas. Para las 15:00 de la tarde, la policía cerró el certamen y los artistas Tang Song y Ciao Lu prendieron fuego a sus propias obras artísticas. La exhibición se abrió dos veces más. Para más información consultar: LAGO, Francesca (1999), “The Avant-Garde Has Its Moment of Glory”, Time Magazine, vol. 154, n 12, pp. 98-99.
} 
al descontento de los ciudadanos con la corrupción política del gobierno de Deng Xiaoping y en defensa de la libertad de prensa y deseos de reforma (Rodríguez, 1990, pp. 134-135).

\section{China's New Art post-1989}

En este desarrollo, siguiendo en la primera etapa de exposiciones sobre el arte chino contemporáneo en el panorama internacional, encontramos la exposición China's New Art post-1989 celebrada del 3 al 25 de febrero de 1993 en la galería Hanart TZ Gallery de Hong Kong [1]. Ésta se presentaba como una importante retrospectiva sobre las últimas tendencias del arte chino y además, siguiendo las palabras del comisario Oscar Ho, la muestra no pretendía ser una contribución o complementación a la importante muestra China/Avant-garde celebrada en Beijing en 1989, a pesar de que las dos compartían la idea de plantear una retrospectiva sobre el arte experimental chino [2]. El comisario explicó cómo aquella primera suponía un vago reconocimiento oficial del nuevo arte chino de una manera temporal, por lo que la presentada en Hong Kong debía dar una imagen alternativa a la primera (Tsong-Zung y Xianting, 1993, pp. 8-9).

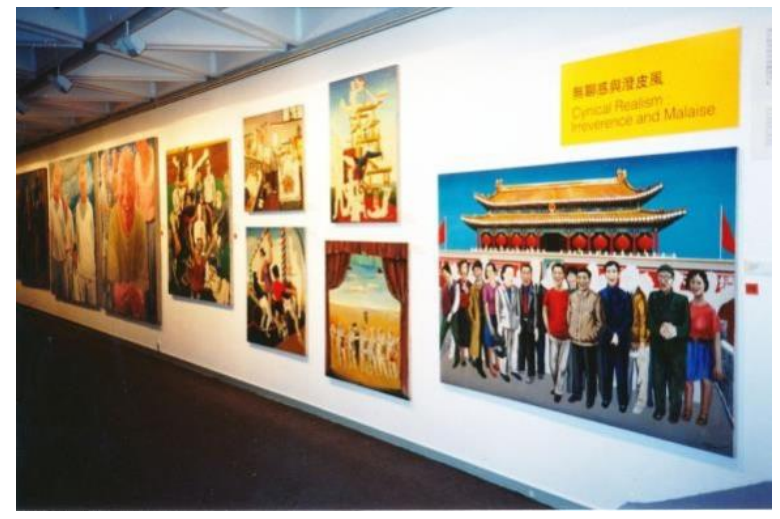

1. Fotografía de la exposición China's New Art post-1989 celebrada en Hong Kong en 1993. 


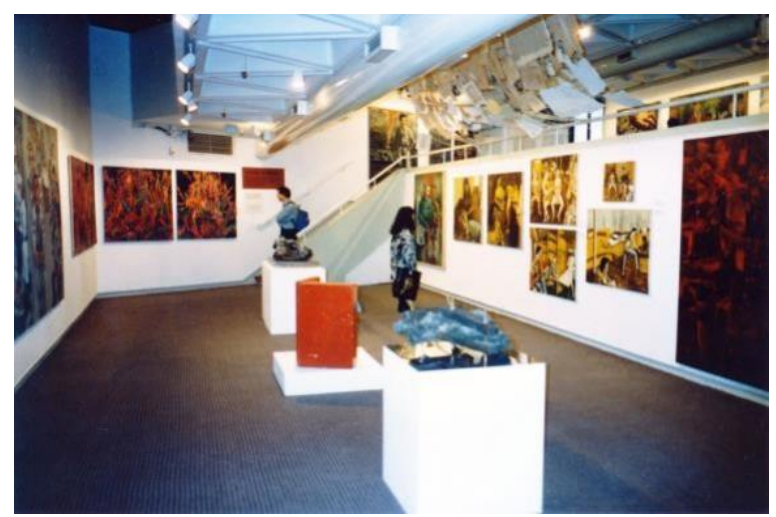

2. Fotografía de la exposición China's New Art post-1989 celebrada en Hong Kong en 1993.

El cambio geográfico y cultural de Beijing a Hong Kong, influía en la perspectiva política de la exposición; ya que su principal objetivo era el de realizar una retrospectiva de artistas experimentales chinos fuera del alcance administrativo del gobierno comunista, pero sin salir del contexto chino. Es decir, los comisarios llevaron a cabo una importante labor de contexto para dar a conocer el arte último del país. Por esta razón, la exposición incluyó en su título el año 1989 ya que según los comisarios “en 1989, China entra en los noventa" (Txong-Zung y Xianting, 1993, p.1).

De esta forma, la muestra se convirtió una de las más influyentes de los años noventa, estando en gira a lo largo de siete años y consolidando a numerosos artistas chinos en el mercado del arte internacional (Erickson, 2002, p.110). Pero, además, tanto en la exposición China's New Art post1989, como en los demás certámenes colectivos de la primera mitad de los noventa, se formalizó una manera similar de presentar el contexto artístico chino. Ejemplo de ello son los catálogos que se publicaron donde lo común era tratar los trabajos artísticos de los artistas relacionándolos con el contexto político y sociológico del país, recalcando el discurso de un desarrollo histórico que partía de un arte arraigado en las tradiciones orientales milenarias hacia una modernidad occidentalizada (Erickson, 2002, p.110).

\section{Inside Out: New Chinese Art}

Entrando en la segunda fase o "segunda ola de interés" de Occidente por el arte experimental o contemporáneo chino, tenemos la exhibición Inside Out: New Chinese Art celebrada entre 1998- 1999, siendo uno de los ejemplos más resaltados. Y es que supuso la mayor muestra realizada hasta entonces en Estados Unidos sobre el tema. No obstante, es necesario señalar que para finales de la década de los noventa, ya se había constituido un mercado de arte contemporáneo chino estable en Occidente, sobre todo en Norteamérica y especialmente a través de las galerías de Nueva York. 
Asimismo, también eran cada vez más frecuentes las exhibiciones colectivas e individuales sobre artistas chinos y eran más abundantes los ensayos académicos de especialistas y textos de críticos sobre el tema (Chiu, 2003, pp.8-21).

Como novedad, Inside Out: New Chinese Art fue la primera gran exhibición en presentar el arte producido por artistas contemporáneos de China, Taiwan y Hong Kong; tanto de aquellos que trabajaban dentro del país, como quienes emigraron al oeste a finales de los ochenta, disminuyendo la previa narración sociológica en el discurso expositivo. El evento fue organizado por la Asia Society Museum de Nueva York y el San Francisco Museum of Modern Art y fue presentado desde el 15 de septiembre de 1998 hasta el 2 de febrero de 1999.

Cabe recordar que la Asia Society Museum, fundada en 1956 por la colaboración de John D. Rockefeller III, es una organización no lucrativa que toma como objetivo la expansión y difusión del mundo asiático al público occidental general [3]. En la actualidad, tiene diversos centros en los Estados Unidos, siendo el más importante de ellos el ubicado en Nueva York, en el cual se incluye el museo que entre otras muestras, exhibe la colección Rockefeller de arte asiático. Por lo tanto, el espacio también se convertía en un elemento elocuente en el mensaje de la exposición, ya que el principal propósito, en palabras del comisario Gao Minglu, era "enriquecer la percepción y la comprensión del público occidental sobre del arte contemporáneo chino, tanto de una forma visual como conceptual" (Gao, 1998, pp.15-40). De esta manera, se intentó explorar la compleja relación existente entre las específicas tensiones culturales y los grandes cambios socioeconómicos vividos en China en la era postmoderna, de ahí que conceptos como globalización y transnacionalización estuvieran presentes en su discurso 9 .

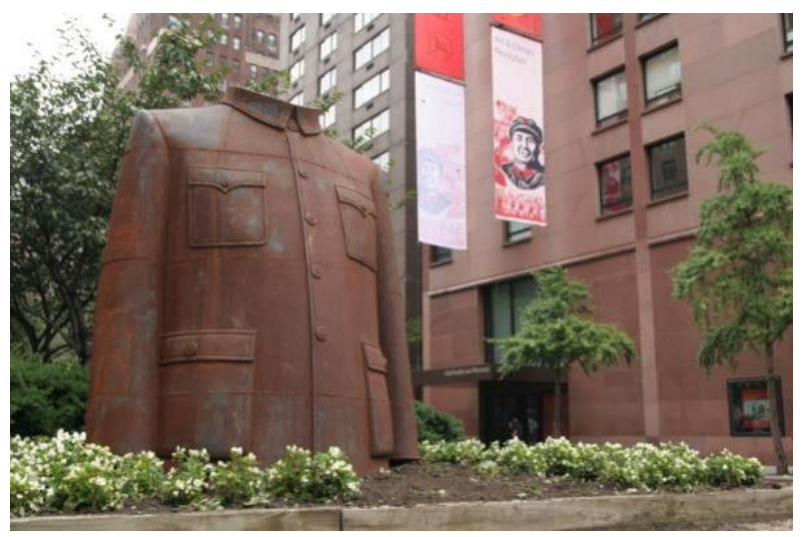

3 Fotografía de la Asia Society Museum de Nueva York.

\footnotetext{
${ }^{9}$ New Chinese Art. Inside out. Recuperado de http://sites.asiasociety.org/arts/insideout/introduction.html (Consulta: 22/02/2018)
} 
En este proceso de apertura y visibilidad en Occidente de la escena artística china, es significativo que el comisario de la muestra fuera Gao Minglu, académico y comisario de las exposiciones más influyentes sobre el arte contemporáneo chino, como la célebre China/Avant- garde. Por lo que es importante reconocer la relevancia que han tenido ciertos autores, comisarios y especialistas en la manera de mostrar tal panorama artístico y de historiar la materia para la $\operatorname{posteridad}^{10}$.

En la exhibición, no se mostraron el tipo de obras a las que hasta entonces había estado acostumbrado el público occidental, tales como la pintura de tinta tradicional o los que seguían la estética del Realismo Socialista. Al contrario, Inside Out: New Chinese Art mostró trabajos de artistas que habían integrado plenamente los lenguajes occidentales vigentes que, para sorpresa del público, eran semejantes a los locales en cuanto a sus formas y discursos (Del Campo, 2013, p. 146). Participaron alrededor de 60 artistas del momento de China, Taiwán y Hong Kong, con diferentes inquietudes y maneras de trabajar, y la exhibición se organizó a través de categorías o temas sociales y artísticos ligados con la realidad china. Estos eran Arte y contexto: caligrafía y significado donde se analizaba el peso que había tenido el arte de la tinta en el mundo artístico chino y su proceso de modernización ${ }^{11}[4]$, temas sociales como Identidad cultural y cambio; Consumismo; Cuestiones familiares: cambio de valores y comentarios sociales [5] y por primera vez las Cuestiones de Género, siendo éste un tema complicado por tratarse desde una perspectiva epistemológica occidental.

4 Fotografía de la instalación Book from the sky (1987-91) del artista Xu Bing presentada en la exhibición.

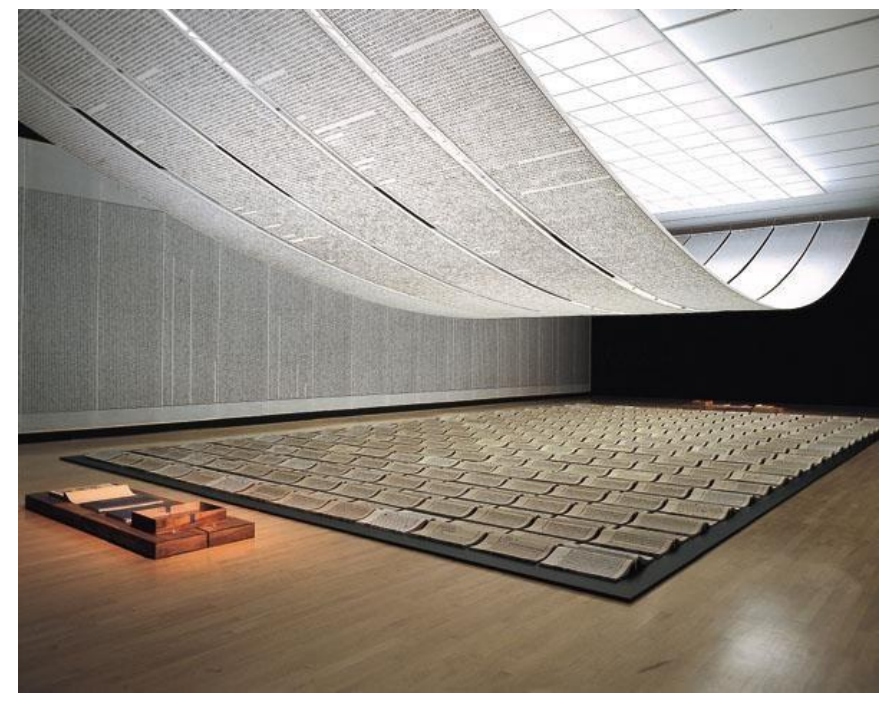

\footnotetext{
${ }^{10}$ LIU, Wei. (2011). http://en.cafa.com.cn/gao-minglu.html (Consulta: 22/02/2018)

${ }^{11}$ Para más información consular: HONG, Lu (2012). "The Formation and Development of New Ink Art in China. Recuperado de http://www.mplusmatters.hk/inkart/paper_topic2.php?l=en (Consulta: 19/12/2017)
} 


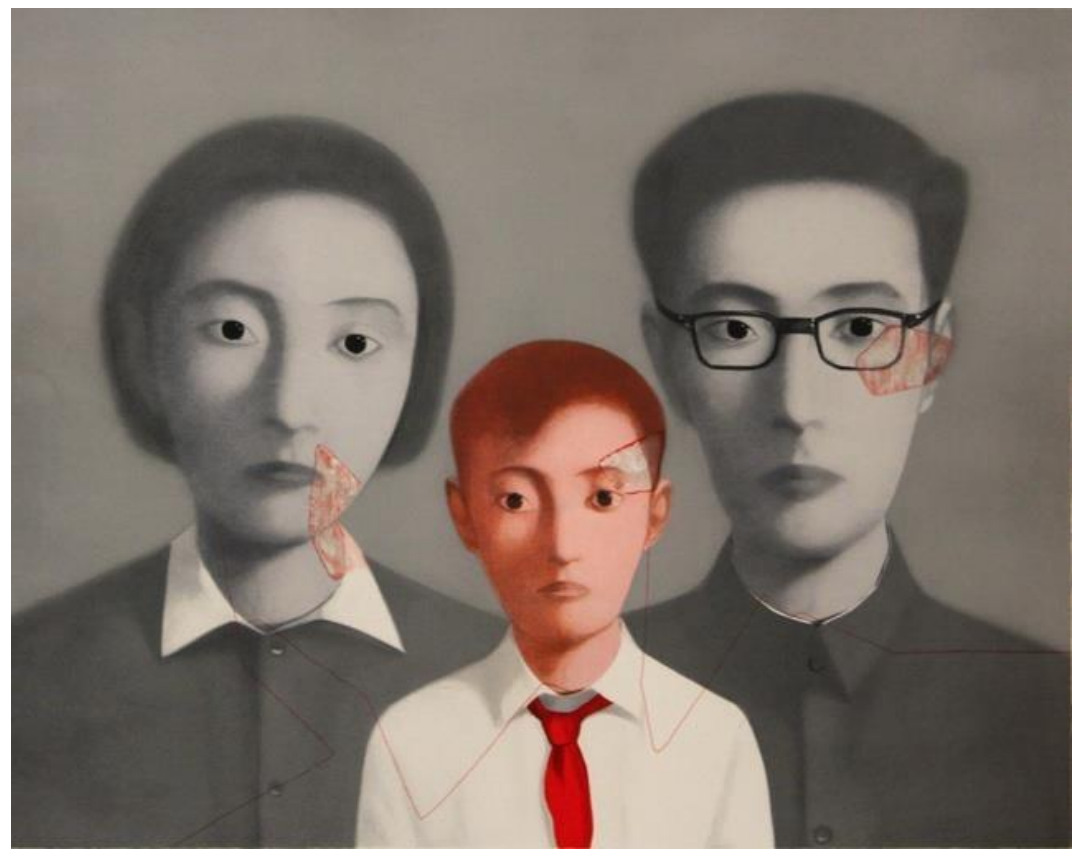

5 Obra Bloodline: Family portrait (1994) del artista Zhang Xiaogang presente en la exposición.

El catálogo de la exposición también mostraba un carácter diferente en comparación con las muestras de la primera etapa. En él se planteaban, a través de diferentes grupos temáticos, contenidos con un carácter y perspectiva diferentes, siendo notable la visión poscolonialista. Estas agrupaciones tenían los títulos de Hacía una modernidad transnacional; El eco de la cultura china en el exterior; Vanguardias post-ideológicas; Modernidad-Postmodernidad; Más allá del Reino Medio; De la élite al hombre pequeño; Desarrollo del arte contemporáneo en Taiwán y Desarrollo del arte en Hong Kong.

Otra distinción de la exposición era que a pesar de que la mayoría de los artistas presentados eran parte del llamado "85 Art New Wave movement", se introdujeron artistas de nuevas generaciones y con diferentes intereses además de creadores también de Hong Kong o de Taiwán, como ya se ha indicado, abriendo un nuevo debate sobre la viabilidad de considerar como unidad a artistas de diferentes contextos sociales (Erickson, 2002, p.108). Asimismo, muchos de estos autores, al no haber estado presentes en las exhibiciones anteriores, eran por entonces desconocidos para el público occidental, entrando rápidamente en el mercado internacional del arte (Del Campo, 2013, p.134).

Por último, la exhibición ayudó a presentar otra imagen del arte contemporáneo chino no visto hasta entonces, en el que resultaba patente que los artistas orientales se identificaban con las 
corrientes artísticas vigentes en el mundo Occidental o global, desmitificando así el carácter exótico de muchas de las exposiciones colectivas previas.

\section{Conclusiones}

Como hemos visto, el caso de la exposición Inside Out: New Chinese Art es elocuente en la tarea de contextualizar la entrada del arte chino contemporáneo en el panorama artístico occidental. Y es que en el reducido marco temporal de los años noventa, resultó perceptible el gran cambio que se produjo en la configuración de las exposiciones que trataban el arte contemporáneo chino tanto en sus características narrativas, en su modo de presentación, en la selección de artistas, en el formato expositivo, en su agrupación temática y en otros matices. Y es que, actualmente, y con una ligera distancia temporal, es palpable cómo aquellas primeras e importantes exposiciones marcaron la dirección en la forma narrativa y en el discurso histórico del arte chino contemporáneo.

Así, por ejemplo, haciendo una comparación entre los dos casos estudiados, la exposición de China's New Art post-1989 se articulaba en torno a unas categorías discursivas muy cerradas, de cierto corte formalista y con una perspectiva puramente sociológica, configurando el recorrido de la exposición a través de distintos estilos o temáticas artísticas. En Inside Out: New Chinese Art en cambio, las categorías que integraban el discurso eran más abiertas e incluían cuestiones de género e identidad. Este cambio de percepción se entiende como un intento por parte de los criterios curatoriales por introducir nuevas metodologías y acercarse a la mentalidad occidental y el individualismo de los artistas.

Por otra parte, es importante recalcar el influyente papel que han jugado los catálogos que se realizaban con motivo de estas exposiciones de los años noventa, convirtiéndose hasta ahora en medio y fuente esencial de difusión para el conocimiento del arte experimental chino en Occidente. En la mayoría de ellos, se incluían numerosos artículos que examinaban el contexto y la historia de China tratando de narrar el proceso que vivió el horizonte artístico desde el arte tradicional de tinta hasta la actualidad.

No obstante, es necesario tener en cuenta cómo muchos de los investigadores o especialistas de estos textos que realizaron la tarea de historiar un periodo artístico concreto y legitimar numerosos artistas, han sido un número reducido de autores y comisarios chinos muy concretos y, por otro lado, especialistas occidentales formados en sus propios países y desde una perspectiva muy concreta. Por ello, esta unifocalidad en sus lecturas, ha repercutido en errores o inexactitudes metodológicas como por ejemplo considerar el arte chino contemporáneo como mero reflejo de la 
realidad socioeconómica que vivió el país con la llegada del capitalismo. Siendo ésta una lectura simplista, lleva a su vez a entender o narrar el arte o la historia del arte chino, desde una perspectiva exclusivamente occidental (Del Campo, 2013, p. 154).

\section{Referencias bibliográficas}

CERVIÑO, Mariana Eva (2015), "Desigualdad y diferencia en el discurso del arte global", Estudios sobre las Culturas Contemporáneas, vol. 21, n 41, pp. 9-39.

CHIU, Melissa (2003). Transexperience and Chinese experimental art, 1990-2000. Sydney: University of Western Sydney.

DEL CAMPO, Eva Fernández (2013), “El arte contemporáneo de China en el proceso de mundialización”, Orientando, n 2, pp. 123-154.

ERICKSON, Britta (2002), "The reception in the West of experimental Mainland Chinese art of the 1990s”, Reinterpretations. Guangzhou: Guangzhou Museum of Art, pp. 105-112.

GAO, Minglu (ed.) (1998). Inside Out: New Chinese Art. University of California Press: Berkeley.

GLADSTON, Paul (2014). Contemporary Chinese Art: A Critical History. Reaktion Books: Islington.

HONG, Lu (2012). The Formation and Development of New Ink Art in China. Recuperado de http://www.mplusmatters.hk/inkart/paper_topic2.php?l=en (Consulta: 19/12/2017)

KOCH, Franziska (2011), “China' on Display for European Audiences? The Making of an Early Travelling Exhibition of Contemporary Chinese Art: China Avantgarde (Berlin/1993)", Transcultural Studies, n $^{\circ}$, pp. 66-139.

LAGO, Francesca (1999), “The Avant-Garde Has Its Moment of Glory”, Time Magazine, vol. 154, $\mathrm{n}^{\mathrm{o}} 12$, pp. $98-99$.

LAGO, Francesca (2000), "Chinese Art at the Venice Biennale: 1.”, en CLARK, John (ed.). Chinese Art at the end of the Millennium. Hong Kong: New Art Media Limited, pp. 158-166.

LIU, Wei. (2011). Gao Minglu. Recuperado de http://en.cafa.com.cn/gao-minglu.html (Consulta: 22/02/2018)

New Chinese Art. Inside out. Recuperado de http://sites.asiasociety.org/arts/insideout/introduction.html (Consulta: 22/02/2018)

RODRÍGUEZ, María Teresa (1990), "El movimiento de mayo de 1989 en China”, Estudios de Asia y África, vol. 25, n 1 , pp. 134-152. 
La entrada del arte chino contemporáneo en Occidente. Inside Out: New Chinese Art, un caso de estudio

TSONG-ZUNG, Chang y XIANTING, Li (1993). China's New Art, Post-1989. Hanart TZ Gallery: Hong Kong. 